\section{GW23-e2605 OILIOIANGXIN INHIBITS THE DEVELOPMENT OF CARDIAC HYPERTROPHY, REMODELLING, AND DYSFUNCTION DURING 4 WEEKS OF PRESSURE OVERLOAD IN MICE}

doi:10.1136/heartjnl-2012-302920a.59

Y Zou, L Lin, Y Ye, J Wei, N Zhou, Y Liang, H Gong, L Li, J Wu, Y Li, Z Jia, Y Wu, J Zhou, J Ge, Y Zou. Shanghai Institute of Cardiovascular Diseases, Zhongshan Hospital and Institutes of Biomedical Sciences, Fudan University, Shanghai, China

Objectives Qiliqiangxin (OL), a traditional Chinese medicine, has been used in the treatment of chronic heart failure. However, whether OL can benefit cardiac remodelling in the hypertensive state is unknown. We here examined the effects of $\mathrm{OL}$ on the development of cardiac hypertrophy through comparing those of losartan in C57BL/6 mice underlying transverse aorta constriction for 4 weeks.

Methods $\mathrm{OL}$ and losartan were administrated at $0.6 \mathrm{mg}$ and $13.4 \mathrm{mg} \mathrm{kg}$, respectively. Cardiac hypertrophy, function, and remodelling were evaluated by echocardiography, catheterisation, histology, and examination of specific gene expression and ERK phosphorylation. Cardiac apoptosis, autophagy, tumour necrosis factor $\alpha$ /insulin-like growth factor-1, and angiotensin II type 1 receptor expression and especially the proliferation of cardiomyocytes and phosphorylation of ErbB receptors were examined in vivo to elucidate the mechanisms.

Results Transverse aorta constriction for 2 weeks resulted in a significant cardiac hypertrophy, which was significantly suppressed by either OL or losartan treatment. At 4 weeks after transverse aorta constriction, although the development of cardiac dysfunction and remodelling and the increases in apoptosis, autophagy, tumour necrosis factor $\alpha /$ insulin-like growth factor-1, and angiotensin II type 1 receptor expression were abrogated comparably between $\mathrm{OL}$ and losartan treatments, OL, but not losartan, enhanced proliferation of cardiomyocytes, which was paralleled with dowregulation of CCAAT/enhancer-binding protein $\beta$, upregulation of $\mathrm{CBP} / \mathrm{p} 300$-interacting transactivator with ED-rich carboxy-terminal domain 4, and increases in ErbB2 and ErbB4 phosphorylation. Furthermore, inhibition of either ErbB2 or CBP/ p300-interacting transactivator with ED-rich carboxy-terminal domain 4 abolished the cardiac protective effects of OL.

Conclusions $\mathrm{QL}$ inhibits myocardial inflammation and cardiomyocyte death and promotes cardiomyocyte proliferation, leading to an ameliorated cardiac remodelling and function in a mouse model of 
pressure overload. The possible mechanisms may involve inhibition of angiotensin II type 1 receptor and activation of ErbB receptors. 